\title{
Effectiveness of Toothpaste Containing REFIX Technology against Dentin Hypersensitivity: A Randomized Clinical Study
}

\author{
Fabiano V Vilhena ${ }^{1}$, Mackeler R Polassi ${ }^{2}$, Eloisa AC Paloco ${ }^{3}$, Roberta CB Alonso ${ }^{4}$, Ricardo D Guiraldo ${ }^{5}$, \\ Paulo HP D’Alpino ${ }^{6}$
}

\begin{abstract}
Aim: This clinical study aimed to evaluate the efficacy of a toothpaste containing a proprietary REFIX technology (Regenerador + Sensitive DentalClean, Rabbit Corp) against dentin hypersensitivity.

Materials and methods: Fifty-three volunteers who fulfilled the inclusion criteria and signed the consent form were included. They were examined for dentin hypersensitivity. The participants received a 1-second blast of air, and the tooth sensitivity, from 0 to 10 , was immediately evaluated using a visual analog scale (VAS). Then, the participants brushed their teeth with the multifunctional toothpaste, and dentin hypersensitivity was tested a second time using the same scale. The participants continued to use the toothpaste three times a day for 1 week, after which dentin hypersensitivity was recorded for the third time. Data were statistically analyzed using analysis of variance (ANOVA) and Tukey's test ( $\alpha=0.05$ ). Results: The mean patient age was 40 years, and $70 \%$ of the 53 subjects were female. There was a significant reduction in dentin hypersensitivity immediately after using the toothpaste and after 1 week. The baseline mean patient-reported pain score was severe $(6.5 \pm 2.4)$. Immediately after the first use of the toothpaste, the mean reported pain significantly decreased to mild pain $(2.5 \pm 2.5)(p<0.05)$. After 1 week of consistent use of the toothpaste, the pain score reduced significantly $(0.7 \pm 1.2)(p<0.05)$, and most participants reported no pain, demonstrating the effectiveness of the REFIX technology against dentin hypersensitivity.

Conclusion: This clinical trial shows that the use of the phosphate-based desensitizing toothpaste containing REFIX technology significantly reduces dentin hypersensitivity after 1 week of consistent use.

Clinical significance: The absence of pain, a desired clinical condition in patients with dentin hypersensitivity, was reached with the use of desensitizing toothpaste containing REFIX technology after 1 week of use. Such condition positively impacts quality of life, providing a healthier daily routine for patients.

Keywords: Clinical trial, Dentin hypersensitivity, Desensitizing, Toothpaste.

The Journal of Contemporary Dental Practice (2020): 10.5005/jp-journals-10024-2847
\end{abstract}

\section{INTRODUCTION}

Dentin hypersensitivity is a clinical condition characterized by acute, transitory, well-localized pain derived from dentinal exposure, which is caused by chemical, volatile, thermal, tactile, or osmotic stimuli, and is not related to other dental defects or pathologies. ${ }^{1-3}$ It affects most of the world population, causing pain and discomfort, in some way reducing the quality of life. ${ }^{4}$ The prevalence of dentin hypersensitivity is $3-57 \%$ in the general adult population. ${ }^{5-7}$ Considering the cases of patients with periodontal problems alone, this prevalence increases up to $98 \% .^{8}$ Despite the variations in prevalence, dentin hypersensitivity occurs more frequently in adults, mostly between 20 years and 40 years of age, peaking at the end of the third decade of life. ${ }^{9}$ Changes in eating habits and stress-related disorders, among other conditions, contribute to the exposure of the dentinal tubules, leading to pain. ${ }^{10}$

Fluoride is effective for protecting dental structures, not only reducing enamel dissolution and improving remineralization, but also treating dentin hypersensitivity by occlusion of the exposed dentin tubules. ${ }^{11,12}$ The main mode of action of fluorine is topical, especially delivered by toothpastes. ${ }^{13}$ Fluoride dentifrices are universally accessible and are affordable oral care products. Toothpastes containing active ingredients against dentin hypersensitivity are the first choice in oral care. ${ }^{8}$ However, toothbrushing with desensitizing products can be difficult most of the time because of persistent or regularly recurrent acute pain (as most results are observed after 4 weeks), leading to
${ }^{1}$ Trials, Research and Development, Inc.

2,6Biotechnology and Innovation in Health Program, Anhanguera University of São Paulo (UNIAN-SP), São Paulo, Brazil

3,5 Department of Restorative Dentistry, School of Dentistry, University North of Paraná-UNOPAR, Londrina, Paraná, Brazil

${ }^{4}$ Department of Dentistry, School of Dentistry, Metropolitan University of Santos, Santos, São Paulo, Brazil; University of Mogi das Cruzes, Mogi das Cruzes, São Paulo, Brazil

Corresponding Author: Paulo HP D'Alpino, Biotechnology and Innovation in Health Program, Anhanguera University of São Paulo (UNIAN-SP), São Paulo, Brazil, Phone: +55 11 35128412, e-mail: paulodalpino@gmail.com

How to cite this article: Vilhena FV, Polassi MR, Paloco EAC, et al. Effectiveness of Toothpaste Containing REFIX Technology against Dentin Hypersensitivity: A Randomized Clinical Study. J Contemp Dent Pract 2020;21(6):609-614.

Source of support: FAPESP 2015/50615-7

Conflict of interest: None

the accumulation of dental plaque in the sensitive areas. ${ }^{7}$ This fact favors the increased risk of caries, gingivitis, and ultimately alveolar bone loss. ${ }^{7}$ These clinical characteristics are considered the main signs of the periodontal disease. ${ }^{14}$ Faced with this problem, researchers and oral care companies are seeking to maintain and improve the clinical effectiveness of fluoride-containing toothpastes by developing new products with promising claims 
of blocking the neural activity in pulp nerves such as potassium. ${ }^{15}$ However, there are several criticisms of the true effectiveness of potassium for treating dentin hypersensitivity, ${ }^{15}$ as studies proving its effectiveness are based on controversial animal models. ${ }^{16-18}$

Dentin hypersensitivity appears to be treated more effectively and consistently when the exposed dentin tubules are occluded, reducing dentin permeability. ${ }^{19}$ For this purpose, biomimetic agents are added to toothpaste formulations and are known as fluoride boosters. ${ }^{20}$ These agents act as calcium and fluoride carriers inside the dental enamel, being more effective as remineralizing agents, as these boosters favor an increase in the size of hydroxyapatite crystals, becoming less soluble and porous. ${ }^{21}$ In dentin, boosters may improve the precipitation of an obliterating mineral layer onto the dentinal tubule openings and/or within the tubules, preventing intratubular fluid movement, ${ }^{22}$ and relieving the dentin sensitivity caused by this fluid movement. ${ }^{2,15}$ There is no agreement on the effectiveness of the over-the-counter products recommended for use in this clinical condition. In this manner, comparative studies have demonstrated that desensitizing toothpastes have varied impacts on the blockage of dentinal fluid movement, which seems to be reflected in the effectiveness of these products for treating dentin hypersensitivity. ${ }^{23}$

Recently, a proprietary technology, REFIX, has been developed. This technology was added to a fluoride-containing toothpaste and other oral care products in association with tetrasodium pyrophosphate and silica. According to the manufacturer, this association favors the formation of a fluoridated apatite and the deposition of silicate, which is also incorporated deep into the hydroxyapatite and onto the open dentinal tubules. These ionic changes ultimately reduce hydroxyapatite solubility, decrease dentinal fluid movement, and improve its mechanical properties, and thus are effective for protecting against dentin hypersensitivity. The purpose of the present study was to evaluate clinically the efficacy of a toothpaste containing this new proprietary technology for reducing dentin hypersensitivity. The research hypothesis was that the use of this toothpaste would reduce the pain reported by patients with dentin hypersensitivity.

\section{Materials and Methods}

\section{Experimental Design}

The present study is a one-week, single-center, single-group, open clinical treatment trial. The experimental unit was patients with dentin hypersensitivity. The intervention proposed was toothbrushing performed by the patient using the toothpaste with REFIX technology for 1 week. The response variable was the evaluation of pain provided by scores according to the visual analog scale (VAS). The factors in analysis were based on evaluation times: (1) baseline: before treatment; (2) immediately after the first use; and (3) after 1 week of consistent use (three times a day). This experimental design for testing a desensitizing product was based on previous studies. ${ }^{24,25}$

\section{Ethical Considerations}

The clinical protocol was approved by The Research Ethics Committee of Universidade Norte do Paraná, Brazil (protocol $3.440 .120 / 2019$, approved clinical trial protocol RBR-36dr7t). This clinical trial was conducted according to Resolution 466/2012 of the Brazilian National Health Council/Ministry of Health, and the Declaration of Helsinki (1964) and its later amendments. All subjects received detailed information about the study and were instructed to sign an written informed consent form before study enrollment.

\section{Patient Selection}

For the present clinical study, 62 patients from private dental practices in São Paulo, São José dos Campos, Londrina, and Bauru (Brazil) presenting dentin hypersensitivity were evaluated for eligibility (Flowchart 1). To be included in the study, subjects had to be between 18 years and 70 years old; be in good health, with no history of allergies to dentifrice ingredients (mainly preservatives and flavorings); present at least one tooth with dentin exposure caused by dental abrasion, erosion, abfraction, or gingival recession; and report tooth hypersensitivity after air blasting stimulus with a minimum score 2 at the baseline examination. ${ }^{26}$ Patient flowchart of this clinical trial is presented in Flowchart 1.

Patients with orthodontic appliances, with moderate or advanced active periodontal disease, with tumors of the soft or hard oral tissues, and pregnant women were excluded from this clinical study. Patients who used analgesics or any other medication that could interfere or potentially mask pain sensation (such as antihistamines, sedatives, anticonvulsants, antidepressants, anti-inflammatory drugs, tranquillizers, or daily analgesics) and patients who routinely used desensitizing toothpaste or any other desensitizing agent were also excluded. In addition, we excluded from the study subjects reporting hypersensitivity in teeth exhibiting enamel cracks or defects, cavitated caries, fractures, mobility, extensive or defective restorations, prosthetic crown or veneer, or pulp involvement. Patients with neuromuscular disorders and other disorders impeditive to perform toothbrushing and patients who are mentally unstable were also excluded. Of the 62 patients selected initially, eight were excluded due to severe active periodontal disease and one was excluded due to age $(<18$ years old); 53 were included in the study.

\section{Clinical Procedures}

The selected patients received information on the etiology of dentin hypersensitivity (identifying predisposing factors such as acid diet, excessive toothbrushing, occlusal trauma, gingival recession) and all instructions for proper oral hygiene to reduce gingiva trauma and to increase plaque control efficiency, as well as dietary advice

Flowchart 1: Patients were preselected by their dentists regarding inclusion and exclusion criteria. Only patients known for dentin hypersensitivity were recruited for the clinical trial

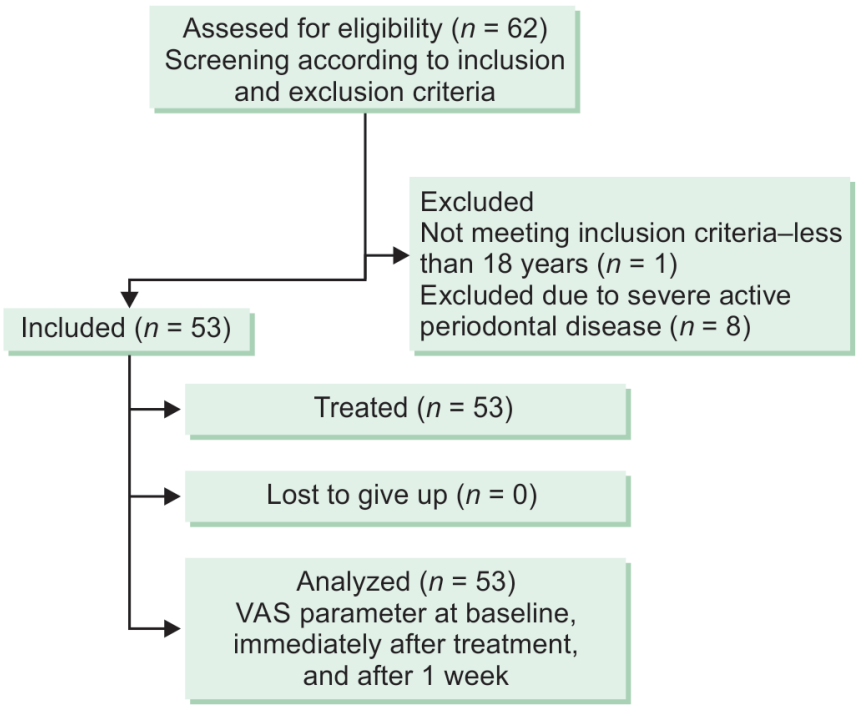


for dental erosion control and caries prevention. The participants were then instructed to brush their teeth three times a day using the dentifrice containing REFIX technology (Regenerador + Sensitive DentalClean, Rabbit Corp, Londrina, PR, Brazil). Each patient received a kit containing an extra-soft toothbrush, the dentifrice, and use instructions. Subjects were instructed to use only their assigned toothpaste, toothbrush, and to discontinue all other oral hygiene practices. Subjects were instructed to brush their teeth three times a day (morning, afternoon, and evening) for at least $90 \mathrm{~s}$ each time.

To evaluate the pain due to air blast hypersensitivity, a 1-second application of air from a three-way syringe handpiece was directed to the exposed dentin surface at a distance of approximately $1 \mathrm{~cm}$. The participants were instructed to point to the pain intensity on a 100-mm VAS, which ranges from no pain (score 0) to worst conceivable pain (score 10). ${ }^{27}$ The VAS pain score was recorded at baseline (before treatment), immediately after use of the desensitizing toothpaste, and after 1 week of consistent use. Immediately after the air stimulus, the participants rated the intensity at which they considered their pain. For participants who reported no or little pain relief after the 1-week treatment, a high-fluoride concentration varnish was applied to the sensitive tooth and the toothpaste containing the REFIX technology was provided for continued use.

\section{Statistical Analysis}

The data were statistically analyzed using repeated-measures analysis of variance (ANOVA) and Tukey's post hoc test, with a significance of $5 \%$, using PAST, version $3.22 .{ }^{28}$

\section{Results}

The mean patient age was 40 years, and $70 \%$ of the 53 participants were female. Patient dropouts were not observed in the present study. Table 1 shows the mean pain level according to the evaluation period; Figure 1 shows the distributional characteristics of the data.

Table 1: Mean score results (standard deviation) based on visual analog scale pain score as a function of the evaluation time

\begin{tabular}{llll}
\hline Group & Baseline & Immediate & After 1 week \\
\hline Mean score & $6.5(2.4) \mathrm{A}$ & $2.5(2.5) \mathrm{B}$ & $0.7(1.2) \mathrm{C}$ \\
\hline
\end{tabular}

Means followed by distinct letters $(A, B$, and $C)$ are significant $(p<0.05)$, $N=53$

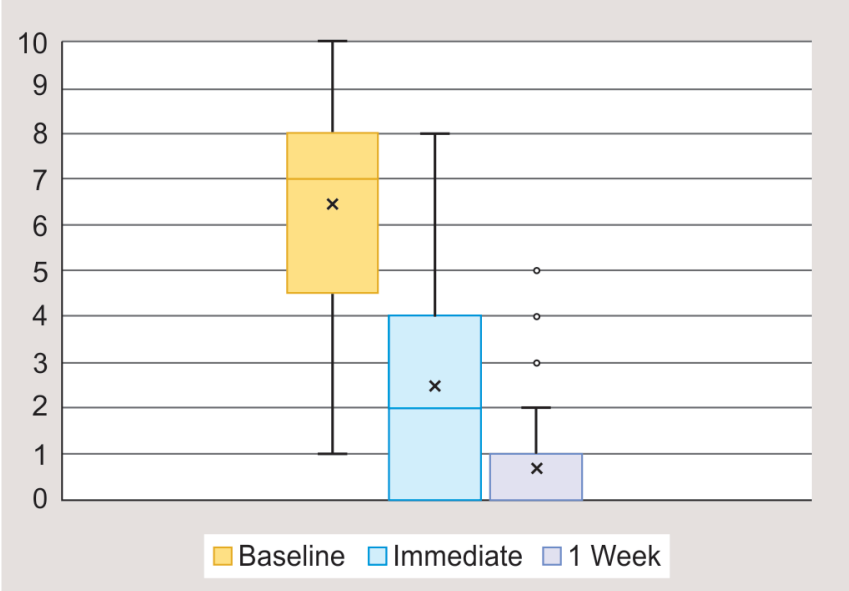

Fig. 1: Boxplot graphic of the recorded pain score by visual analog scale comparing the evaluation periods (baseline - control, immediately after use, and after 1 week). Significant differences were observed between the three periods
A significant reduction in pain level was observed immediately after the first brushing with the product $(p<0.05)$. The mean pain level reduced by $62.5 \%$ after the first use (from 6.5 to 2.5 ) (Table 1). After 7 days of consistent use, the patient-reported pain level also significantly reduced to $0.7(p<0.05)$, representing a mean decrease of $88.3 \%$ as compared to the baseline (Table 1 ).

Figure 2 shows a comparison between mean VAS scores as a function of pain relief immediately and after 1 week of continuous use of the REFIX-containing toothpaste: Immediately after the application of the product, $30.2 \%$ of the participants reported complete remission of the sensitivity (score 0 ), $45.3 \%$ reported a decrease in sensitivity levels of $\geq 50 \%$, and $24.5 \%$ reported little or no pain relief. After 1-week use of the toothpaste, $30.2 \%$ of the participants reported complete remission of the sensitivity (score 0 ), $30.2 \%$ reported maintained pain remission (no pain return), 34\% reported sensitivity reduction of $\geq 50 \%$, and only $5.7 \%$ reported little or no pain relief (Fig. 2).

\section{Discussion}

Clinicians have recommended the use of different professional and home therapeutic agents for treating dentin hypersensitivity. ${ }^{3}$ Most studies that evaluated an "at-home" desensitizing therapy reported a more effective result after 3 to 4 weeks of use. ${ }^{7}$ Conversely, the results of the present clinical trial demonstrated that the great majority of participants reported pain relief after 1 week of use (Figs 1 and 2). The effectiveness of the REFIX-containing toothpaste was proved by the statistical analysis which demonstrated that there was a significant difference $(p<0.05)$ in the mean scores of pain relief considering the patient distribution when the different evaluation times were compared (between baseline and the immediate means; and comparing immediate and 1-week findings) (Table 1). In this manner, the research hypothesis, which anticipated that the use of this toothpaste would reduce the pain reported by patients with dentin hypersensitivity, was accepted.

The reasons for this effectiveness are based on its formulation and mode of action. The toothpaste with the REFIX technology is an

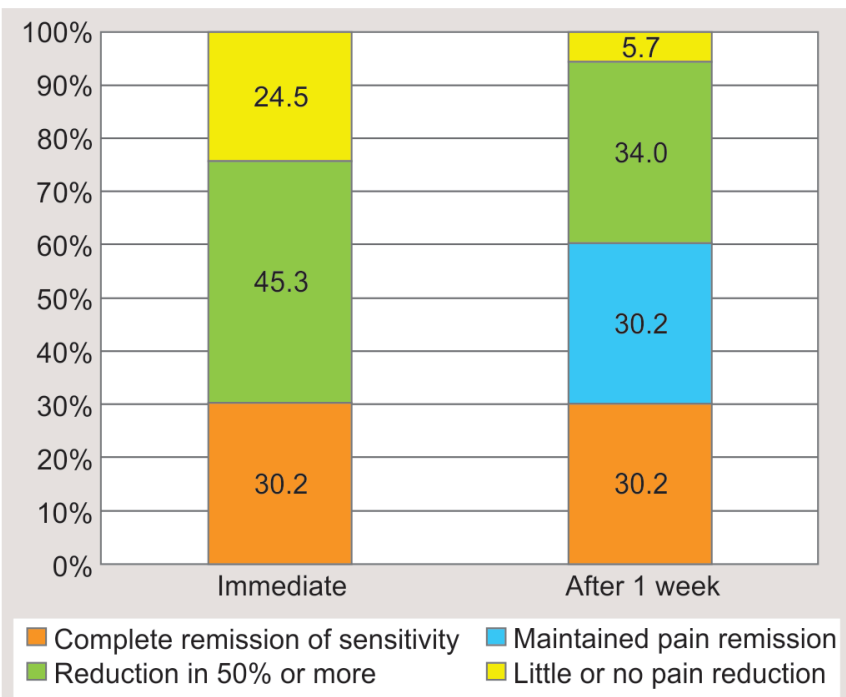

Fig. 2: Patient distribution (in percentage) according to pain relief as a function of the evaluation time (immediately after use and after 1 week of continuous use of the REFIX-containing toothpaste). Data are presented as mean percentage 
acidic toothpaste, containing silica, 1450 ppm fluoride (as sodium fluoride), and tetrasodium pyrophosphate. Based on manufacturer's technical profile, it comprises a multifunctional phosphate-based oral care product in an acidified phosphate/fluoride stable complex, in the presence of saliva in the oral cavity. In this manner, new minerals containing calcium/phosphate/fluorine are generated, ${ }^{29}$ which tends to superficially remineralize not only the dental tissues, but also the subsuperficial layers, thus reducing the dentin hypersensitivity. It can be speculated that crystal-like deposits are formed within the tubule lumen.

It is true that remineralization of dental enamel is a slow process, so it does not seem possible to completely occur immediately after only one application. Conversely, the multifunctional phosphatebased acidic toothpaste containing REFIX technology comprises a stabilized phosphate/fluoride complex. When in contact with the oral environment, this complex interacts with the dental structures. Tetrasodium pyrophosphate maintains ionic calcium because it penetrates the demineralized tissues and/or precipitates in the form of loosely bound calcium fluoride. ${ }^{30}$ Silicates have been used in toothpaste formulations, allowing the formation of calcium silicate, augmenting the natural mineralization processes of saliva via hydroxyapatite nucleation and mineral formation. ${ }^{31}$ The study of substituting phosphate ions $\left(\mathrm{PO}_{4}\right)$ with silicate ions $\left(\mathrm{SiO}_{4}\right)$ in hydroxyapatite structures is recent. ${ }^{32}$ In an in vitro study, ${ }^{33}$ it was evaluated the remineralizing effects of calcium silicate deposition on the properties of acid-eroded dental surfaces. The authors demonstrated that calcium silicate was able to provide a protective effect by inducing the formation of hydroxyapatite after its deposition onto the eroded surfaces. ${ }^{33}$

It could be speculated that this acidic toothpaste could negatively jeopardize the remineralization process in a previously demineralized dentin, or the use of an acidic toothpaste could increase dentin hypersensitivity due to additional erosive action. However, the remineralizing and anticaries effectiveness of acidified dentifrices are well documented in the literature, ${ }^{21}$ although its effect on dentin sensitivity is poorly discussed. Besides, even with the vast literature on the effect of fluoride-containing toothpastes, the exact mechanism of fluoride compounds associated with other ingredients in sealing the tubules remains unclear and could be related to the $\mathrm{pH}$ condition. Despite the lack of information, the mechanism by which acidic toothpaste decalcifies the dentin has been reported. ${ }^{34,35}$ The acidic $\mathrm{pH}$ favors the increase in the ionized calcium concentration that exceeds the solubility product constant for calcium fluoride, after which the calcium ions precipitate on and inside the tubules. It has also been proven that fluoridecontaining oral care products that are slightly acidic present no erosive potential. ${ }^{36} \mathrm{~A}$ previous study ${ }^{37}$ pointed out that, depending on the $\mathrm{pH}$, different ionized stages of $\mathrm{H}_{3} \mathrm{PO}_{4}$ deprotonation can be observed. It has also been highlighted that $100 \%$ of $\mathrm{H}_{2} \mathrm{PO}_{4}{ }^{-}$is available to interact with calcium ions at $\mathrm{pH} 4.5$, which is similar to that of the REFIX-containing toothpaste, leading to a wide variety of calcium phosphate phases.

Based on previous studies, phosphates and pyrophosphates appear to mediate the formation of less soluble mineral deposits. ${ }^{38-40}$ In this manner, it can be speculated that the acidic property of the fluoride-containing REFIX-based toothpaste can etch the intertubular and peritubular dentin, releasing ionized calcium ions into the tubular fluid, which can react with the active ingredients of the toothpaste. In this manner, the increased enamel solubility at low $\mathrm{pH}$ leads to an increase in calcium concentrations and thus facilitates the precipitation of calcium fluoride. ${ }^{35}$ Phosphate-mediated calcium fluoride and calcium silicate crystals can be formed and then deposited in the dentinal tubules. Thus, these deposited crystals can reduce the functional area of the dentinal tubules by partially occluding the tubule orifices, reducing the fluid flow, and consequently, dentin hypersensitivity. The clinical effectiveness of these materials may partially depend on the dissolution resistance or solubility of those globules. ${ }^{41}$

It is true that superficial tubule occlusion alone could be easily dissolved by daily toothbrushing and toothbrushing after consuming acidic foods and beverages, leading to short-term desensitizing effects. The dissolution of the deposits could be more due to the direct effect of the acidic food and beverages rather than only the toothbrush effect. Conversely, the results of the present clinical trial demonstrate that REFIX technology is effective for reducing dentin hypersensitivity after an initial application, with a $62.5 \%$ decrease in the baseline pain level. The effectiveness of the REFIXcontaining toothpaste against dentin hypersensitivity continued after for up to 1 week of use, with a mean decrease of $88.3 \%$. The reasons that explain the ability of the REFIX-containing toothpaste to provide pain relief are in accordance with the literature, in which faster and less soluble, long-lasting deposits of calcium fluoride crystals occluding the tubules are observed with a toothpaste with a remarkably lower $\mathrm{pH}$. In this case, due to the high concentration of acids, which favors an increased availability of calcium through dental tissue dissolution, calcium fluoride crystals tend to form quickly, that is, in $<20$ seconds. ${ }^{35}$ The presence of abrasives in the toothpaste may also have played a role in occluding the dentinal tubules. This helps to explain the results of the present study, in which the reported pain in one out of three participants decreased from severe to painless immediately after use of the toothpaste.

In addition to the reduced dentin hypersensitivity reported here, the toothpaste with REFIX technology has also demonstrated protective effects on enamel regarding caries and erosion. ${ }^{42}$ The effectiveness of $1450 \mathrm{ppm}$ fluoride-containing toothpastes containing different active remineralization agents after cariogenic challenge with $\mathrm{pH}$ cycling was investigated. ${ }^{42}$ The authors found out that the enamel subsurface was more effectively remineralized, among other effective commercial products, when the REFIX-containing toothpaste was used. Oversaturated phosphate concentrations in the formulation of the toothpaste seem to contribute to the increased calcium and phosphate on the demineralized enamel surface, thus increasing the resistance to the demineralizing process in the presence of fluoride ions.

The fact that this 1-week study was performed to evaluate the effectiveness of the desensitizing toothpaste containing REFIX technology deserves explanation. Oral health problems, most notably dentin sensitivity, which invariably lead to painful sensitivity at different levels, can affect an individual's well-being, leading the patients to live with constant discomfort, culminating in physical and emotional pain. In this manner, the desirable impact on clinical outcomes would be the fast relief in the pain, which was proven as previously described. The favorable outcomes of the toothpaste to provide relief from dentin sensitivity can be also attributed to the adherence of the subjects to the proposed treatment plan. Moreover, it is important to highlight that one of the given recommendations was to discontinue all other oral hygiene practices. Although it was not calculated, the compliance rate in short-term therapy is usually found to be high (between $70 \%$ and $80 \%),{ }^{43}$ having a major effect on the treatment outcomes 
and positive clinical consequences obtained with the use of the toothpaste tested.

Considering the limitations of the present clinical trial, the lack of a negative control for comparative reasons seems to have affected the interpretation of the results. For ethical reasons, a negative control was not included. In addition, experimental designs for testing desensitizing products in a single-group, open clinical treatment trial have been previously reported. ${ }^{24,25}$ The other limitations include the subjectivity of VAS for recognizing pain and the patients' knowledge as participants of the clinical trial. Compliance bias was not influenced by the participants' responses, as they had no personal relation with the investigator, and no incentives were offered to the participants. The short duration of the clinical trial could be another point of contention. It is true that the lack of a longer duration of follow-up for confirming the efficacy of this desensitizing product could be considered a limitation of this study. Conversely, the analysis of the rapid relief of pain arising from exposed dentin in response to different stimuli following the use of this pain-relieving product warrants evaluation. This could be attributed to the inclusion criterion "report pain of minimum score 2 at the baseline examination of the tooth hypersensitivity after air blasting stimulus" to verify the efficacy of the treatment. This can introduce bias to the results, as the chance of completely stopping the pain after one application of the toothpaste is higher. Conversely, considering that this is the first clinical trial to examine the effectiveness of this desensitizing product and the first description of a treatment with REFIX technologycontaining toothpaste, no direct comparisons can be made between the present and/or previous results.

\section{ConcLusion}

This clinical trial demonstrates that toothbrushing associated with the desensitizing toothpaste containing REFIX technology significantly reduces dentin hypersensitivity immediately after the first use, with a reduction after 1 week of consistent use. Further comparative studies may be required to confirm the effectiveness and the mechanism of the toothpaste tested.

\section{References}

1. Davari A, Ataei E, Assarzadeh H. Dentin hypersensitivity: Etiology, diagnosis and treatment; A literature review. J Dent (Shiraz) 2013;14(3):136-145.

2. Hu ML, Zheng G, Zhang YD, et al. Effect of desensitizing toothpastes on dentine hypersensitivity: a systematic review and meta-analysis. J Dent 2018;75:12-21. DOI: 10.1016/j.jdent.2018.05.012.

3. George AA, Muruppel AM, Sudeep S, et al. A comparative evaluation of the effectiveness of three different modalities in occluding dentinal tubules: an in vitro study. J Contemp Dent Pract 2019;20(4):454-459. DOI: 10.5005/jp-journals-10024-2538.

4. Bekes K, John MT, Schaller HG, et al. Oral health-related quality of life in patients seeking care for dentin hypersensitivity. J Oral Rehabil 2009;36(1):45-51. DOI: 10.1111/j.1365-2842.2008.01901.x.

5. Splieth $\mathrm{CH}$, Tachou A. Epidemiology of dentin hypersensitivity. Clin Oral Investig 2013;17(Suppl 1):S3-S8. DOI: 10.1007/s00784-012-0889-8.

6. Cunha-Cruz J, Wataha JC, Heaton LJ, et al. The prevalence of dentin hypersensitivity in general dental practices in the northwest United states. J Am Dent Assoc 2013;144(3):288-296. DOI: 10.14219/jada. archive.2013.0116.

7. Miglani S, Aggarwal V, Ahuja B. Dentin hypersensitivity: Recent trends in management. J Conserv Dent 2010;13(4):218-224. DOI: 10.4103/0972-0707.73385.

8. Rosing CK, Fiorini T, Liberman DN, et al. Dentine hypersensitivity: analysis of self-care products. Braz Oral Res 2009;23(Suppl 1):56-63. DOI: 0.1590/S1806-83242009000500009.
9. Bartold PM. Dentinal hypersensitivity: a review. Aust Dent J 2006;51(3):212-218. DOI: 10.1111/j.1834-7819.2006.tb00431.x.

10. Meurman JH, Vesterinen $\mathrm{M}$. Wine, alcohol, and oral health, with special emphasis on dental erosion. Quintessence Int 2000;31(10):729-733.

11. Petersson LG. The role of fluoride in the preventive management of dentin hypersensitivity and root caries. Clin Oral Investig 2013;17(Suppl 1):S63-S71. DOI: 10.1007/s00784-012-0916-9.

12. Casals E, Boukpessi T, McQueen CM, et al. Anticaries potential of commercial dentifrices as determined by fluoridation and remineralization efficiency. J Contemp Dent Pract 2007;8(7):1-10. DOI: $10.5005 /$ jcdp-8-7-1.

13. Cury JA, Tenuta LM. Evidence-based recommendation on toothpaste use. Braz Oral Res 2014;28(spe):1-7. DOI: 10.1590/S180683242014.50000001 .

14. Cochran DL. Inflammation and bone loss in periodontal disease. J Periodontol 2008;79(Suppl 8):1569-1576. DOI: 10.1902/ jop.2008.080233.

15. Addy M, West NX. The role of toothpaste in the aetiology and treatment of dentine hypersensitivity. Monogr Oral Sci 2013;23:75-87. DOI: 10.1159/000350477.

16. Kim S. Hypersensitive teeth: Desensitization of pulpal sensory nerves. J Endod 1986;12(10):482-485. DOI: 10.1016/S0099-2399(86)80203-5.

17. Peacock JM, Orchardson R. Effects of potassium ions on action potential conduction in A- and C-fibers of rat spinal nerves. J Dent Res 1995;74(2):634-641. DOI: 10.1177/00220345950740020301.

18. Peacock JM, Orchardson R. Action potential conduction block of nerves in vitro by potassium citrate, potassium tartrate and potassium oxalate. J Clin Periodontol 1999;26(1):33-37. DOI: 10.1034/j.1600051x.1999.260106.x.

19. Ganss C, Klimek J, Starck C. Quantitative analysis of the impact of the organic matrix on the fluoride effect on erosion progression in human dentine using longitudinal microradiography. Arch Oral Biol 2004;49(11):931-935. DOI: 10.1016/j.archoralbio.2004.05.010.

20. Philip N. State of the art enamel remineralization systems: the next frontier in caries management. Caries Res 2019;53(3):284-295. DOI: 10.1159/000493031.

21. Delbem ACB, Pessan JP. Alternatives to enhance the anticaries effects of fluoride. In: Coelho Leal S, Takeshita EM. Pediatric Restorative Dentistry. Cham: Springer International Publishing; 2019. pp. 75-92.

22. West N, Seong J, Davies M. Dentine hypersensitivity. Monogr Oral Sci 2014;25:108-122. DOI: 10.1159/000360749.

23. Joao-Souza SH, Scaramucci T, Buhler Borges A, et al. Influence of desensitizing and anti-erosive toothpastes on dentine permeability: an in vitro study. J Dent 2019. 103176. DOI: 10.1016/j.jdent.2019. 07.014 .

24. Bekes K, Heinzelmann K, Lettner S, et al. Efficacy of desensitizing products containing $8 \%$ arginine and calcium carbonate for hypersensitivity relief in $\mathrm{MIH}$-affected molars: An 8-week clinical study. Clin Oral Investig 2017;21(7):2311-2317. DOI: 10.1007/s00784016-2024-8.

25. Ayad F, Ayad N, Vazquez J, et al. Use of a toothpaste containing $8 \%$ arginine and calcium carbonate for immediate and lasting relief of dentin hypersensitivity: a simple and effective in-office procedure. Am J Dent 2018;31(3):135-140.

26. Schiff T, Delgado E, Zhang YP, et al. Clinical evaluation of the efficacy of an in-office desensitizing paste containing $8 \%$ arginine and calcium carbonate in providing instant and lasting relief of dentin hypersensitivity. Am J Dent 2009;22:8A-15A.

27. Delgado DA, Lambert BS, Boutris N, et al. Validation of digital visual analog scale pain scoring with a traditional paper-based visual analog scale in adults. J Am Acad Orthop Surg Glob Res Rev 2018;2(3):e088. DOI: 10.5435/JAAOSGlobal-D-17-00088.

28. Hammer $\varnothing$, Harper DAT, Ryan PD. PAST: paleontological statistics software package for education and data analysis. Palaeontol Electron 2001;4(1):9.

29. Pirca K, Balbin-Sedano G, Romero-Tapia P, et al. Remineralizing effect of casein phosphopeptide-amorphous calcium phosphate and sodium fluoride on artificial tooth enamel erosion: an in vitro 
study. J Contemp Dent Pract 2019;20(11):1254-1259. DOI: 10.5005/ jp-journals-10024-2710.

30. Mello SV, Arvanitidou E, Stranick MA, et al. Mode of action studies of a new desensitizing mouthwash containing $0.8 \%$ arginine, PVM/ MA copolymer, pyrophosphates, and $0.05 \%$ sodium fluoride. J Dent 2013;41(Suppl 1):S12-S19. DOI: 10.1016/j.jdent.2012.11.001.

31. Moreno EC, Kresak M, Zahradnik RT. Fluoridated hydroxyapatite solubility and caries formation. Nature 1974;247(5435):64-65. DOI: 10.1038/247064a0.

32. Silva HM, Mateescu M, Ponche A, et al. Surface transformation of silicon-doped hydroxyapatite immersed in culture medium under dynamic and static conditions. Colloids Surf B Biointerfaces 2010;75(1):349-355. DOI: 10.1016/j.colsurfb.2009.09.009.

33. Parker AS, Patel AN, Al Botros R, et al. Measurement of the efficacy of calcium silicate for the protection and repair of dental enamel. J Dent 2014;42(Suppl 1):S21-S29. DOI: 10.1016/S0300-5712(14) 50004-8.

34. Greenhill JD, Pashley DH. The effects of desensitizing agents on the hydraulic conductance of human dentin in vitro. J Dent Res 1981;60(3):686-698. DOI: 10.1177/00220345810600030401.

35. Larsen MJ, Richards A. The influence of saliva on the formation of calcium fluoride-like material on human dental enamel. Caries Res 2001;35(1):57-60. DOI: 10.1159/000047432.

36. Lussi A, Hellwig E. Risk assessment and causal preventive measures. Monogr Oral Sci 2014;25:220-229. DOI: 10.1159/000360612.
37. Meyer F, Amaechi BT, Fabritius HO, et al. Overview of calcium phosphates used in biomimetic oral care. Open Dent J 2018;12(1): 406-423. DOI: 10.2174/1874210601812010406.

38. Lagerlof F, Saxegaard E, Barkvoll P, et al. Effects of inorganic orthophosphate and pyrophosphate on dissolution of calcium fluoride in water. J Dent Res 1988;67(2):447-449. DOI: $10.1177 / 00220345880670020201$.

39. Mellberg JR, Petrou ID, Deutchman M, et al. The effects of $1 \%$ pyrophosphate and $0.02 \%$ sodium fluoride on artificial caries lesions in vivo. J Dent Res 1988;67(12):1461-1465. DOI: 10.1177/00220345880670120401.

40. Ibsen CJS, Birkedal H. Pyrophosphate-inhibition of apatite formation studied by in situ X-ray diffraction. Minerals 2018;6(2):1-9. DOI: $10.3390 /$ min8020065.

41. Wang Z, Sa Y, Sauro S, et al. Effect of desensitising toothpastes on dentinal tubule occlusion: a dentine permeability measurement and SEM in vitro study. J Dent 2010;38(5):400-410. DOI: 10.1016/ j.jdent.2010.01.007.

42. Tomaz PLS, Sousa LA, Aguiar KF, et al. Effects of 1450-ppm fluoridecontaining toothpastes associated with boosters on the ename remineralization and surface roughness after cariogenic challenge. Eur J Dent 2020;14(1):161-170. DOI: 10.1055/s-0040-1705072.

43. Jin J, Sklar GE, Min Sen OV, et al. Factors affecting therapeutic compliance: a review from the patient's perspective. Ther Clin Risk Manag 2008;4(1):269-286. DOI: 10.2147/tcrm.s1458. 